\title{
Análisis del modelo de desarrollo organizacional de las empresas públicas anexas al gobierno autónomo descentralizado de Manabí, para promover proyectos de desarrollo socio económico en la provincia de Manabí
}

\section{Analysis of the organizational development model of the public companies attached to the decentralized autonomous government of Manabí, to promote socio-economic development projects in the province of Manabí}

Ec Mariana Bustamante Chong

Ing. Byron Caamaño Guerrero

Universidad Estatal del Sur de Manabí, Ecuador

Ing. César Antonio Bustamante Chong

Universidad Ecotec, Ecuador

Autor para correspondencia: byron.caamano@unesum.edu.ec, mariana.bustamante@unesum.edu.ec, cbustamante@ecotec.edu.ec

Fecha de recepción: 30 de Agosto de 2016 - Fecha de aceptación: 25 de Octubre de 2016

Resumen: El presente Trabajo de Investigación sobre Administración Pública, tuvo como objetivo "Analizar la incidencia del modelo de desarrollo organizacional de las empresas públicas anexas al gobierno autónomo descentralizado de Manabí en la promoción de proyectos de desarrollo económico en la provincia de Manabí para perfeccionarlas y aplicarlas adecuadamente, las técnicas y los instrumentos utilizados, se ciñeron a través de Técnicas de observación y se elaboraron para observar el comportamiento de los representantes de los cantones, parroquias y comunas de la provincia de Manabí,en las encuestas se emplearon cuestionarios dirigidos a representantes de los cantones, parroquias y comunas de la provincia de Manabí, empleados y autoridades de las empresas públicas anexas al gobierno autónomo descentralizado de Manabí para saber si las actividades que realizan dichos empleados y autoridades han ayudado en la promoción de proyectos de desarrollo socio económico de la provincia de Manabí, y además conocer las actividades que tienen los empleados para la promoción de dichos proyectos. Se realizaron preguntas abiertas y cerradas, el trabajo de investigación se desarrolló a través de una metodología participativa con la responsabilidad de los investigadores y las unidades de observación, la metodología empleada consto de Método Hermenéutico, Método Analítico Sintético y la Estadística; en este estudio se plantea la hipótesis: "El modelo de desarrollo organizacional de las empresas públicas que se realiza en la provincia de Manabí limita el desarrollo de la promoción de proyectos de desarrollo socio económico en la provincia de Manabí”; es así que El fundamento teórico aborda tres temas principales: en el primero se trata el Desarrollo Organizacional de las empresas anexas al Gobierno Provincial de Manabí; en el segundo tema se trata del Gobierno autónomo descentralizado provincial de Manabí; el tercer tema es La Empresa Pública en Manabí y finalmente un cuarto tema en el cual se hace referencia a la Gestión de Proyectos de Desarrollo Socioeconómico. Fruto de todo el proceso. La conclusión primordial es: Se considera que las empresas publicas tanto de Patronato Provincial, como Manabí Construye realizan una labor eficiente en la gestión de proyectos de desarrollo socioeconómico de la población Manabita, claro que los proyectos que estas empresas ejecutan no son autogenerados sino que surgen de otros organismos y grupos sociales es así que estas empresas solo intervienen en la parte ejecutoria. Mientras 
que la recomendación es: Es preciso que las empresas Patronato Provincial y Manabí Construye impriman su eficacia en la gestión de nuevos proyectos los que sean autogenerados desde el diagnostico hasta la solución de problemas.

Palabras claves: empresas públicas; proyectos de desarrollo socioeconómico; gapm, manabí construye; patronato provincial

Abstract: The present investigation on Public Administration, aimed to "analyze the impact model of organizational development of related public companies to self-government decentralized Manabi in promoting economic development projects in the province of Manabi to perfect and properly apply , techniques and instruments used, girded by observation techniques and were prepared to observe the behavior of the representatives of the cantons, parishes and communes of the province of Manabi, surveys questionnaires were used for representatives of the cantons, parishes and communes of the province of Manabi, employees and authorities of the attached public companies to self-government decentralized Manabi to know if their activities such employees and authorities have helped in promoting projects of socio-economic development of the province Manabi, and also about the activities that have employees to promote such projects. open and closed questions were conducted, the research was developed through a participatory approach with the responsibility of researchers and observation units, the methodology consisted of hermeneutical method, Analytical Method Synthetic and Statistics; in this study the hypothesis arises: "The model of organizational development of public enterprises is carried out in the province of Manabi limits the development of projects promoting socioeconomic development in the province of Manabi"; Thus the theoretical foundation addresses three main issues: the first Organizational Development of businesses related to the Provincial Government of Manabi concerned; the second topic is the decentralized autonomous provincial government of Manabi; The third issue is the public company in Manabi and finally a fourth theme in which reference to Project Management Socio-Economic Development is done. The result of the whole process. The primary conclusion is: It is considered that public companies both Provincial Board, as Manabi Build perform efficient work in project management of socio-economic development of the Manabita population clear that projects these companies are running are not self-generated but arise other agencies and social groups so that these companies only involved in the enforcement part. While the recommendation is that companies must Provincial and Manabi print Build effective in the management of new projects that are self-generated from the diagnosis to troubleshooting.

Key words: public enterprises; economic development projects; gapm, manabí build; provincial

\section{Introducción}

En el marco de la construcción de Constitución Política de la República del Ecuador en el año 2008, la Asamblea Constituyente de Montecristi, realizo importantes cambios en pro de la participación interinstitucional en el desarrollo socioeconómico de las provincias, sin duda uno de los más importantes es el Art. 315 de la Constitución de la República del Ecuador que dispone que el Estado constituya empresa pública para la gestión de sectores estratégicos, la prestación de servicios públicos, el aprovechamiento sustentable de los recursos naturales o de bienes públicos y el desarrollo de otras actividades económicas. ${ }^{1}$

Este artículo guarda concordancia con lo manifestado en el Art. 5. De la Ley Orgánica de Empresas Publicas “los Gobierno Autónomos, están facultados para constituir, organizar, fusionar y liquidar empresas públicas, mediante Ordenanza que permitan la prestación eficiente

\footnotetext{
${ }^{1}$ Constitución de la república del Ecuador, Montecristi 2008
} 
de los servicios públicos que son de su competencia"”2 Es así que el Gobierno Provincial de Manabí conforma seis importantes empresas de servicio público, como son: Manabí Construye, Patronato Provincial, Empresa pública de Administración Vial, Agencia de Desarrollo Provincial de Manabí, CORFAM Corporación Forestal y de Ambiental de Manabí y APIM Agencia de Promoción de Inversión de Manabí.

Estas Empresas Públicas cuentan con autonomía financiera, jurídica y administrativa, pero es importante que en dicho marco se direccionen los resultados hacia la creación de proyectos de desarrollo socioeconómico, de manera autónoma, de tal manera que los pueda realizar a partir de un diagnostico real y puedan realizar su gestión y seguimiento, a fin de impulsar de manera más eficiente el desarrollo socioeconómico de la población de la provincia de Manabí. Con este propósito se ha planteado la presente investigación.

Este documento está estructurado en 11 epígrafes de gran importancia, entre los cuales está el planteamiento del problema, la justificación, los objetivos, las variables e hipótesis, el marco metodológico, el marco o fundamento teórico conceptual, los resultados, conclusiones, recomendaciones y la correspondiente bibliografía.

\section{Desarrollo}

Se sintetiza la problemática principalmente en:

El Patronato Provincial de Manabí es el ente encargado dentro del gobierno autónomo provincial de manejar las políticas y planes dirigidos a los grupos sociales mayormente vulnerables en la provincia, esta entidad intenta desarrollar y definir mecanismos de articulación y corresponsabilidad entre los diferentes actores inmersos en la actividad, intentando lograr una eficiente y oportuna atención de las personas que conforman estos grupos de atención prioritaria.

La empresa pública "Manabí construye" tiene limitantes en el diseño y construcción de la infraestructura de vías en las zonas urbanas y rurales de la provincia debido a la falta de planificación y ejecutividad de anteriores administraciones en las cuales se ha priorizado otros tipos de factores muy ajenos al bienestar colectivo y crecimiento sustentable de la geografía provincial.

Pero es importante conocer si en realidad estas empresas direccionan de manera eficiente sus esfuerzos en el desarrollo socioeconómico de los habitantes de la provincia de Manabí; se debe conocer si estas empresas pueden de forma autónoma diagnosticar problemas, planificar soluciones, condensarlas en un proyecto de desarrollo socioeconómico que sea funcional y factible de cumplimiento y si de la misma manera pueden realizar su seguimiento, es decir si se cuenta con un modelo de desarrollo organizacional que permita estas actividades.

Nos incentivamos a realizar este trabajo de investigación, por las siguientes razones:

La acción de las empresas anexas al Gobierno Provincial Autónomo de Manabí justifican su accionar en el sin número de obras y apoyo a los grupos mayormente vulnerables y con menos representación dentro de la sociedad Manabita.

\footnotetext{
${ }^{2}$ Ley Orgánica de Empresas Públicas del ecuador, Reg. Of. № 48, octubre 16 del 2008
} 
Los proyectos implantados por las empresas públicas del Gobierno Provincial, buscan impulsar el desarrollo en diferentes comunidades tanto en las áreas urbanas como rurales ya sea con el trabajo social y la inversión efectuada en estos sitios alejados de la geografía provincial, con la única prioridad que es el de satisfacer las necesidades del entorno de la comunidad, provocando de esta forma que la comunidad se beneficie y desarrolle.

A través de los modelos organizacionales que se proponen en la presente investigación se trata de sugerir la implementación de un desarrollo integral para el buen vivir, sustentado mediante la estimulación a un proceso de desarrollo tanto a los niveles de alta gerencia, administrativo y con el desarrollo de las comunidades con el fortalecimiento y creación de nuevas capacidades.

Esta investigación sugiere un análisis de los modelos de gestión con la finalidad de proponer directrices que faciliten la ejecución de un modelo de desarrollo socio económico a través del establecimiento de una planificación eficiente y la incorporación de una gestión por resultados que logre un desarrollo integral sustentable que permita la optimización de los recursos económicos y laborales con que cuentan estas empresas anexas al Gobierno Provincial.

Este estudio particularmente, propiciará resultados positivos dirigidos a la comunidad, ya que mediante la propuesta se planteará que se planifique y optimice los recursos y el personal de las empresas públicas de Gobierno Provincial mediante un modelo de desarrollo organizacional eficiente, logrando la inversión en servicio social y en obras prioritarias que permitan mejorar la calidad de vida y el desarrollo humano en los sectores más vulnerables y de atención prioritaria en la Provincia de Manabí

Este trabajo investigativo tiene como Objetivos los siguientes:

\section{Objetivo General}

- Analizar la incidencia del modelo de desarrollo organizacional de las empresas públicas anexas al gobierno autónomo descentralizado de Manabí en la promoción de proyectos de desarrollo económico en la provincia de Manabí para perfeccionarlas y aplicarlas adecuadamente.

\section{Objetivos Específicos}

- Explicar las Características del modelo de desarrollo organizacional de las empresas públicas anexas al gobierno autónomo descentralizado de Manabí en la promoción de proyectos de desarrollo socio económico en la provincia de Manabí.

- Describir la promoción de proyectos de desarrollo socio económico en la provincia de Manabí, por acción del modelo de desarrollo organizacional de las empresas públicas anexas al gobierno autónomo descentralizado de Manabí.

- Construir lineamientos alternativos para mejorar la problemática investigada.

Mediante este trabajo investigativo se pudo demostrar que:

El modelo de desarrollo organizacional de las empresas públicas que se realiza en la provincia de Manabí limita el desarrollo de la promoción de proyectos de desarrollo socio económico en la provincia de Manabí. El trabajo de investigación se desarrolló a través de una 
metodología participativa con la responsabilidad de los investigadores y las unidades de observación, con el:

Método Descriptivo utilizado para representar la realidad del modelo de desarrollo organizacional de las empresas públicas anexas al gobierno autónomo descentralizado de Manabí, que se emplean en dichas Instituciones y describir las actividades para promover proyectos de desarrollo socio económico en la provincia de Manabí. El Método Hermenéutico utilizado para la lectura e interpretación de los diferentes textos para completar y enriquecer el marco teórico de la investigación a realizar.

EI Método Analítico Sintético: utilizado para formular la respectiva investigación teórica, empírica y las conclusiones. Además para analizar la situación actual respecto al modelo de desarrollo organizacional implementado en las empresas públicas anexas al gobierno autónomo descentralizado de Manabí y sus efectos alcanzados. Y la Estadística utilizada para tabular los datos de las encuestas, sistematizarlos, interpretarlos, graficarlos y de acuerdo a ello realizar las conclusiones respectivas

Las técnicas y los instrumentos utilizados, se ciñeron a través de Técnicas de observación y se elaboraron para observar el comportamiento de los representantes de los cantones, parroquias y comunas de la provincia de Manabí

En las encuestas se emplearon cuestionarios dirigidos a representantes de los cantones, parroquias y comunas de la provincia de Manabí, empleados y autoridades de las empresas públicas anexas al gobierno autónomo descentralizado de Manabí para saber si las actividades que realizan dichos empleados y autoridades han ayudado en la promoción de proyectos de desarrollo socio económico de la provincia de Manabí, y además conocer las actividades que tienen los empleados para la promoción de dichos proyectos. Se realizaron preguntas abiertas y cerradas.

En el contexto de la investigación, se realizaron encuestas estructuradas dirigidas a representantes de los cantones, parroquias y comunas de la provincia de Manabí , empleados y autoridades de las empresas públicas anexas al gobierno autónomo descentralizado de Manabí para conocer los procedimientos del modelo de desarrollo organizacional de las empresas publicas anexas al gobierno autónomo descentralizado de Manabí y la existencia o no de la promoción de proyectos de desarrollo socio económico en la provincia de Manabí.

La encuesta aplicada a los empleados y las autoridades de las empresas públicas anexas al gobierno autónomo descentralizado de Manabí, fue para conocer el modelo de promoción de proyectos de desarrollo socio económico en la provincia de Manabí.

Se ha considerado como población de esta investigación a los 32 empleados de la Empresa Pública Manabí Construye, más los 22 del Patronato Provincial de Manabí. Haciendo un total de 54 empleados. Autoridades 2.

La muestra es extraída de la población de 380 representantes de los cantones, parroquias y comunas de la provincia de Manabí encuestados para conocer el nivel de 
promoción de los proyectos de desarrollo socio económico en dicha provincia, la muestra es 105, garantizando un nivel de confianza del 0,95 y un margen de error del 0,5.

$$
\begin{aligned}
& \mathrm{n}=? \\
& \mathrm{P}=50 \% \\
& \mathrm{Q}=50 \% \\
& \mathrm{~N}=380 \\
& \mathrm{Z}=96 \% \\
& \mathrm{e}=5 \%
\end{aligned}
$$

$$
\mathrm{n}=\frac{(0,96)^{2}(0,50)(0,50)(380)}{(0,96)^{2}(0,50)(0,50)+380(0,04)^{2}}
$$$$
87.552
$$$$
\mathrm{n}=\overline{(0.2304)+380(004) 2}
$$

$$
\begin{array}{ll}
\mathrm{n}=\frac{87.552}{0.8384} & \\
\mathrm{n}=104.429 & =105
\end{array}
$$

\section{Marco teórico}

\section{Desarrollo organizacional de las empresas adscritas al gobierno provincial de Manabí}

Todas las empresas que pertenecen al gobierno provincial de Manabí deben tener en claro que la creación, planificación y gestión de proyectos, tienen una importancia y contribución al desarrollo socio económico promoviendo la producción de la provincia enmarcados en los distintos aspectos teniendo la visión y la misión de hacer cumplir las mismas.

\section{Gobierno autónomo descentralizado provincial de Manabí.}

En este se detalla todo lo concerniente al Gobierno Autónomo Descentralizado Provincial de Manabí, qué significa, su organización territorial, los Planes estratégicos, la administración de relaciones públicas, sus fines, competencias, facultades, los servicios públicos a cargo del mismo y su modalidad de gestión.

Como se puede ver todos estos puntos antes mencionados tienen su importancia para el desarrollo adecuado y óptimo del Gobierno Autónomo Descentralizado Provincial de Manabí, y así mismo velar por las gestiones facilitando la buena ejecución de obras en la provincia. 
La organización territorial actual, se la ha dividido de la siguiente forma:

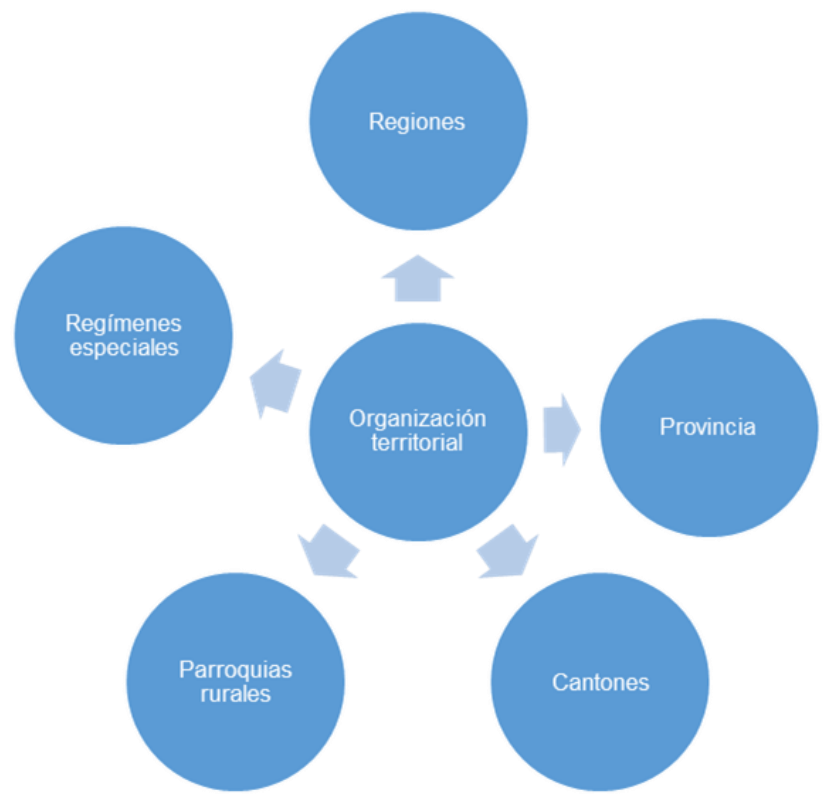

Fuente: Guía Didáctica sobre descentralización y gobiernos autónomos UTPL Elaboración: Los autores.

\section{La Empresa Pública en Manabí.}

Las empresas públicas en Manabí están dedicadas a la producción de bienes y servicios para la venta en el mercado y cuyas operaciones económicas y financieras se encuentran incluidas en el Presupuesto de Egresos de la Federación (control directo) o sólo las relativas a las transferencias de recursos que reciben para apoyar su funcionamiento (control indirecto), su propósito fundamental no es el lucro sino la obtención de objetivos sociales o económicos.

Las empresas públicas tienen poderes públicos pueden ejercer, directa o indirectamente, una influencia dominante en razón de la propiedad, de la participación financiera o de las normas que las rigen.

\section{Gestión de Proyectos de Desarrollo Socioeconómico}

\section{Programas y Proyectos de Desarrollo Socioeconómicos}

Un proyecto de desarrollo socioeconómico es la unidad mínima de asignación de recursos, que a través de un conjunto integrado de procesos y actividades pretende transformar una parcela de la realidad, disminuyendo o eliminando un déficit, o solucionando un problema. Es por tal la importancia de estos proyectos ya que su fin es el mejorar las condiciones de vida en especial de grupos sociales.

Un proyecto de desarrollo socioeconómico debe cumplir las siguientes condiciones: 
$>$ Definir el, o los problemas sociales, que se persigue resolver (especificar cuantitativamente el problema antes de iniciar el proyecto).

$>$ Tener objetivos de impacto claramente definidos (proyectos con objetivos imprecisos no pueden ser evaluados).

$>$ Identificar a la población objetivo a la que está destinada el proyecto (la que teniendo las necesidades no puede satisfacerlas autónomamente vía el mercado).

$>$ Especificar la localización espacial de los beneficiarios.

$>$ Establecer una fecha de comienzo y otra de finalización.

Los proyectos de desarrollo socioeconómico producen y/o distribuyen bienes o servicios (productos), para satisfacer las necesidades de aquellos grupos que no poseen recursos para solventarlas autónomamente, con una caracterización y localización espacio-temporal precisa y acotada. Sus productos se entregan en forma gratuita o a un precio subsidiado.

Un programa socioeconómico es un conjunto de proyecto de desarrollo socioeconómico que persiguen los mismos objetivos, y que por su envergadura no pueden ser asumidos por un único proyecto, como se indicaba anteriormente es la unidad mínima de asignación de recursos, que pueden diferenciarse por trabajar con poblaciones diferentes y/o utilizar distintas estrategias de intervención.

La política social es un conjunto de programas que pretenden alcanzar los mismos fines. Da las orientaciones sobre qué problemas sociales priorizar y define las principales vías y/o límites para la intervención que la política plantea.

Los programas y proyectos de desarrollo socioeconómico, se enmarcan en una política, de la que constituyen su traducción operacional, vía la asignación de recursos que permite su implementación, en el caso en estudio la asignación de dichos recursos debería ser realizada por los departamentos contables de las empresas públicas, derivándola de las ganancias.

Tradicionalmente, los proyectos se definen por la existencia de inversión, esto es, porque se asignan recursos para la adquisición de bienes de capital (terreno, construcciones, equipamiento).

Los programas, alternativamente, supondrían sólo la utilización de recursos para gastos corrientes (por ejemplo, los sueldos y salarios que se requieren para la operación). Hoy existe acuerdo en que los proyectos no se definen por la presencia o ausencia de inversión, sino por ser una unidad organizada de gestión que busca solucionar un problema. Aun cuando la inversión sea inexistente o marginal, todo proyecto puede y debe ser evaluado. 


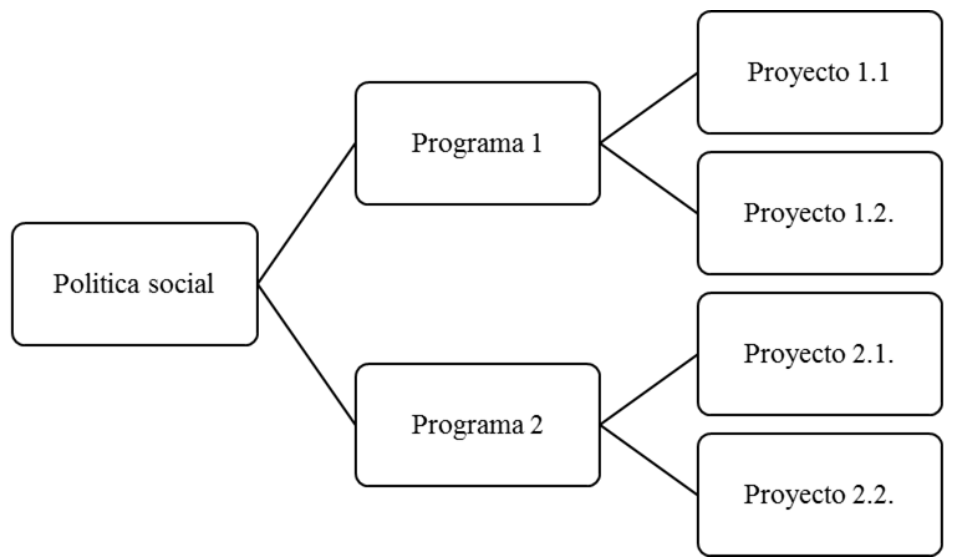

Cuadro: Relación entre Política, Programas y Proyectos de Desarrollo Socioeconómico Fuente: Manual de formulación, evaluación y Monitoreo de Proyectos Sociales

Elaboración: Los autores.

Es decir de la política surgen los lineamientos que definen la formulación de programas y a partir de dichos programas se desarrollan los proyectos de desarrollo socioeconómico.

\section{Recomendaciones}

Es preciso que las empresas Patronato Provincial y Manabí Construye impriman su eficacia en la gestión de nuevos proyectos los que sean autogenerados desde el diagnostico hasta la solución de problemas.

La gerencia Tanto en la empresa Manabí construye como en Patronato Provincial, debe propiciar la autogeneración de proyecto de desarrollo socioeconómico mediante una dirección acertada de toda la empresa.

Se debe realizar una socialización y sensibilización entre los empleados de las empresas publicas Manabí Construye y Patronato Provincial en torno a la importancia de generar de forma autónoma los proyectos de gestión.

Tanto Manabí Construye como Patronato Provincial deben incorpora en su estructura organizacional departamento de creación y gestión de nuevos proyectos de desarrollo socioeconómico destinado al diagnóstico de realidades sociales para la detección de problemas y su solución mediante la gestión de proyectos de desarrollo socioeconómico.

Es precisa que las áreas de la empresa cuenten con lineamientos de procedimientos y actividades para trabajar en equipo con el departamento de creación y gestión de nuevos proyectos de desarrollo socioeconómico.

Las empresas públicas de Patronato Provincial y Manabí construye deben contar con una normativa para la creación y gestión de proyectos de desarrollo socioeconómico en beneficio de la población manabita.

En el departamento de creación y gestión de nuevos proyectos de desarrollo socioeconómico se debe incluir el área de seguimiento y monitoreo de los proyectos de 
desarrollo socio-económico autogenerados. Los empleados de las empresas públicas consideradas en este estudio deben recibir una adecuada capacitación para la creación y gestión de proyectos de desarrollo socioeconómico.

\section{Conclusiones}

Se considera que las empresas publicas tanto de Patronato Provincial, como Manabí Construye realizan una labor eficiente en la gestión de proyectos de desarrollo socioeconómico de la población Manabita, claro que los proyectos que estas empresas ejecutan no son autogenerados sino que surgen de otros organismos y grupos sociales es así que estas empresas solo intervienen en la parte ejecutoria.

Tanto en la empresa Manabí construye como en Patronato Provincial, existe un alto compromiso por parte de la gerencia en torno a la ejecución de proyectos de desarrollo socioeconómico en beneficio de la población, lamentablemente esta gerencia no emplea el mismo compromiso en el desarrollo de proyectos de manera autónoma.

Existe un muy bajo nivel de socialización de la importancia que tendría la gestión autónoma de proyectos de desarrollo socioeconómico en las empresas Patronato Provincial y Manabí Construye.

Las empresas públicas en cuestión no cuentan con un departamento destinado al diagnóstico de realidades sociales sobre las cuales se detecte dificultades y se plasmen proyectos de desarrollo socioeconómico para su solución.

Al no contar con el departamento de creación y gestión de nuevos proyectos de desarrollo socioeconómico las áreas de la empresa tampoco cuentan con lineamiento de procedimientos y actividades para trabajar en equipo con dicho departamento o con los encargados de dicha gestión.

Las empresas públicas de Patronato Provincial y Manabí construye no cuentan con una normativa para la creación y gestión de proyectos de desarrollo socioeconómico en beneficio de la población manabita.

Actualmente la empresa pública de Patronato Provincial y Manabí Construye si realizan seguimiento y monitoreo de los proyectos de desarrollo socio-económico, claro está que esto son solo proyectos ejecutados, más no se trata de proyectos autogenerados.

Los empleados de las empresas públicas consideradas en este estudio no han recibido una adecuada capacitación para la creación y gestión de proyectos de desarrollo socioeconómico.

\section{Bibliografía}

Alberich, Tomas (2010); Perspectivas de la Investigación social; la investigación Social participativa; Valencia; Ed. El Viejo Topo. 
Ambrose, Kenith, (2003); Control Social de Servicios; Ministerio de Bienestar social, GTZ-PMA; Quito; Ed. Abya Yala.

Astorga, A. y Bart Van Der Bijl, (2004); Manual d Diagnóstico Participativo; Quito.

Burgwal, G. y Cuellar, J.C, (2000); Planificación Estratégica y Participativa aplicada a Gobiernos Locales; Quito.

CEPAL (1999); Manual de Formulación y Evaluación de Proyectos Sociales; CEPAL-OEAPROPOSAL; Santiago de Chile.

Constitución de la República del Ecuador, Montecristi 2008

COOTAD: Código Orgánico de Ordenamiento Territorial Autonomías y Descentralización; REG. OF. N 303 de martes 19 de Octubre de 2010; Impreso en Edit. Nacional; Quito.

Cunill Nuria, (1997)Repensando lo público a través de la sociedad, nuevas formas de Gestión Pública y representación social; Caracas; Ed. Nueva Sociedad.

Davis Case D’Arcy, (2009); Herramientas para la comunidad. Conceptos, métodos y herramientas para el diagnóstico, seguimiento y evaluación participativos en el desarrollo campesino; FAO-FTTP-Abya Yala; Quito.

Jara Oscar, (2004); Para sistematizar experiencias; ALFORJA, Costa Rica.

Ley Orgánica de Empresas Públicas; REG. OF. No 48, de octubre 16 de 2009

Martinic, Sergio, (1998); Elementos metodológicos para la sistematización de proyectos de Educación y Acción Social; CIDE; Santiago de Chile.

PNUD, 2003; Informe sobre Desarrollo humano y Participación Popular; Edic. Mundi-Prensa; Madrid.

Romero, J.C, (2000), Sondeo de metodologías participativas en el Ecuador; Corporación OIKOSFTPP; Quito.

Sánez Alvaro, (2002); Estándares para un proceso de desarrollo local; AME- ODEPLANCONAMU-Ministerio del Ambiente-INEC-UNFPA, Quito.

Scrimshaw Susan y Hurtado Elena, (2008); Procedimientos de Asesoría Rápida PARA, para programas de nutrición y atención primaria de salud; UNICEF-UCLA; Los Ángeles.

Secaira Durango, Patricio, (2008); Derecho Administrativo; ed. UTPL; Primera Edición; Loja.

Rebollo, Luis Martin, (2005); Leyes Administrativas; Thomson Arazandi Navarra; $11^{\text {o }}$ edición; Quito. Human Services. 
Navarrete Báez, F. E. (2013). Las Micro, Pequeñas y Medianas Empresas de a Zona Metropolitana de Guadalajara: una perspectiva hacia la gestión de su proceso de toma de decisiones. Revista Electrónica Nova Scientia.Vol 5, num 10, 210 - 236.

Ordóñez Islas, P. A. (2012). Metodología para el diagnóstico organizacional de PYMES (Tesis). México, D. F.: Universidad Nacional Autónoma De México.

Pérez Gorostegui, E. (1994). Economía de la Empresa Aplicada. Madrid: Pirámide.

Porter, M. (2003). Ser competitivo: nuevas aportaciones y conclusiones. Barcelona: DEUSTO S.A.

Rodríguez Valencia, J. ( 2002). Administración de pequeñas y medianas empresas, 5ta Edición. México: Thomson.

Romero, R. (1999). Marketing. Palmir E.I.R.L.

Ross, A. G., Zeballos, J. L., \& Infante, A. (2000). La calidad y la reforma del sector de la salud en América Latina y el Caribe. Revista Panamericana de Salud Pública.vol.8 n.1-2, On-line version ISSN 1680-5348.

Secretaría Nacional de Planificación y Desarrollo. (2012). Plan Nacional de Desarrollo, Plan Nacional para el Buen Vivir 2009-2013: Construyendo un Estado Plurinacional e Intercultural. . Quito: SENPLADES.

Universidad San Martín de Porres. (2012). Organización y gestión de PYMES. Perú: Universidad San Martín de Porres. Facultad de Ciencias Administrativas y Recursos Humanos.

Velasco, C. (9 de Febrero de 2010). La toma de decisiones del emprendedor a cargo de una Pyme. Coyuntura Económica, págs. http://coyunturaeconomica.com/emprendimiento/toma-dedecisiones-pyme. 